Annals of Pure and Applied Mathematics

Vol. 17, No. 2, 2018, 197-202

ISSN: 2279-087X (P), 2279-0888(online)

Published on 2 June 2018

www.researchmathsci.org

DOI: http://dx.doi.org/10.22457/apam.v17n2a5

Annals of

Pure and Applied

Mathematics

\title{
The Minimum Total Edge Dominating Energy of a Graph
}

\section{S. N. Banasode ${ }^{1}$ and Y.M.Umathar ${ }^{2}$}

. 'Department of Mathematics, R. L. Science Institute Belagavi, Karnataka, India

Email: suwarn_nam@yahoo.co.in

${ }^{2}$ Department of Mathematics, K.L.E.Technological University, Hubballi

Karnataka, India.

Corresponding author. Email: yogeeshwar@ bvb.edu

Received 11 April 2018; accepted 2 June 2018

Abstract. The minimum covering energy $E_{C}(G)$ and minimum dominating energy $E_{D}(G)$ of a graph are well known problems of graph theory. Motivated by the work of [1,7] and also by similar works, in this paper, we introduced minimum total edge dominating energy $E_{E d}(G)$ of a Graph is introduced. Minimum total edge dominating energy of a Star Graph and complete graph is computed.

Keywords: Minimum total edge dominating set, minimum total edge dominating matrix, Minimum total edge dominating eigen values, Minimum total edge dominating energy of a graph.

AMS Mathematics Subject Classification (2010): 05C50, 05C69

\section{Introduction}

The concept of energy of a graph was introduced by Gutaman [4], in the year 1978. Let $G$ be a graph with n-vertices and m-edges. Let $A=\left(a_{i j}\right)$ be the adjacency matrix of a graph. Let $\lambda_{1}, \lambda_{2}, \lambda_{3}, \ldots \ldots \ldots \lambda_{n}$ be the eigenvalues of adjacency matrix of a graph $G$. The values are assumed to be in non-decreasing order, that is $\lambda_{1} \geq \lambda_{2} \geq \lambda_{3} \geq$ $\ldots \ldots \ldots \geq \lambda_{n}$. Since $A(G)$ is real and symmetric. Its eigenvalues are real number the energy $E(G)$ of graph is defined to the sum of absolute values of its eigenvalues of graph $G$ that is $E(G)=\sum_{1}^{n}\left|\lambda_{i}\right|$.

Motivated by the research articles, A survey on energy of a graph [8], energies like Distance energy [2], Minimum covering energy [1], Incidence energy [6], Lalpacian energy [5], Domination in graphs [7, 9], and importance of domination number [3], we introduced the minimum total edge dominating energy is defined in this paper and same is obtained for some standard graphs.

\section{Minimum total edge dominating energy}

All graphs considered here are finite, undirected, without loops and multiple edges. Let $G=(V, E)$ be a graph with vertex set $V$ and edge set $E$. 
Let $E=\left\{e, e_{2}, e_{3}, \ldots \ldots \ldots e_{p}\right\}$. A Set $D \subseteq E$ is a total edge dominating set of $G$ if every edge in $E$ is adjacent to some edge in $D$, and edges in $D$ are adjacent. Any total edge dominating set with minimum cardinality is called a minimum total edge dominating set. Let $D$ be minimum total edge dominating set of $G$. The minimum total edge dominating matrix of $G$ is $p \times p$ matrix defined by $A_{E d}(G)=\left(a_{i j}\right)$ where,

$$
a_{i j}=\left\{\begin{array}{l}
1 \text { if } e_{i} \text { and } e_{j} \text { are adjacent } \\
1 \text { if } i=j \text { and } e_{i} \in D \\
0 \text { otherwise }
\end{array}\right.
$$

The characteristic polynomial of $A_{E d}(G)$ is denoted by

$f_{E n}(G, \lambda)=\operatorname{det}\left(\lambda I-A_{E d}(G)\right)$ The minimum total edge dominating eigenvalues of the graph $G$ are the eigenvalues of $A_{E d}(G)$. The matrix $A_{E d}(G)$ is a real and symmetric. Then the eigenvalues of $A_{E d}(G)$ are real numbers and are labeled in non-increasing order $\lambda_{1} \geq \lambda_{2} \geq \cdots \geq \lambda_{p}$ The minimum total edge dominating energy of $G$ is defined as $E_{E d}(G)=\sum_{1}^{p}\left|\lambda_{i}\right|$.

Example 2.1. Let $C_{3}$ be the cycle with 3 -vertices say $v_{1}, v_{2}, v_{3}$ then the minimum total edge dominating set $D=\left\{e_{1}\right\}$ Therefore

$$
A_{E d}\left(C_{3}\right)=\left[\begin{array}{lll}
1 & 1 & 1 \\
1 & 0 & 1 \\
1 & 1 & 0
\end{array}\right]
$$

The characteristic polynomial of $A_{E d}\left(C_{3}\right)$ is $-\lambda^{3}+\lambda^{2}+3 \lambda+1$ and the characteristic equation is $-\lambda^{3}+\lambda^{2}+3 \lambda+1=0$. The minimum total edge dominating eigenvalues are $-1,1+\sqrt{2}$ and $1-\sqrt{2}$. Therefore the minimum total edge dominating energy is give by

$$
E_{E d}\left(c_{3}\right)=|-1| 1+|(1+\sqrt{2})|+|(1-\sqrt{2})|=1+2 \sqrt{2} \approx \mathbf{3 . 8 2 8 4} .
$$

Example 2.2. Let $G$ be the graph with 6-vertices say $v_{1}, v_{2}, v_{3}, v_{4,}, v_{5}, v_{6}$ as shown in the figure 1

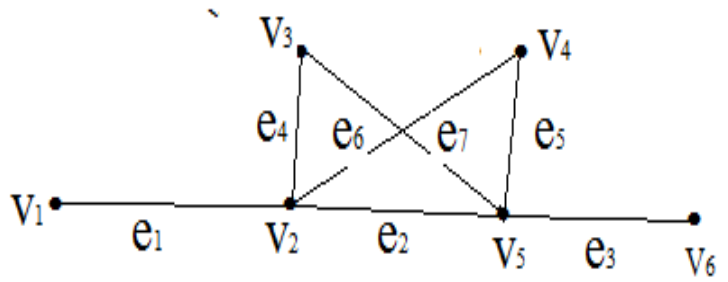

Figure 1: Simple connected graph

Then the minimum total edge dominating set, $D=\left\{e_{2}\right\}$ Therefore

$$
A_{E d}(G)=\left[\begin{array}{lllllll}
0 & 1 & 0 & 1 & 0 & 0 & 1 \\
1 & 1 & 1 & 1 & 1 & 1 & 1 \\
0 & 1 & 0 & 0 & 1 & 1 & 0 \\
1 & 1 & 0 & 0 & 0 & 1 & 1 \\
0 & 1 & 1 & 0 & 0 & 1 & 1 \\
0 & 1 & 1 & 1 & 1 & 0 & 0 \\
1 & 1 & 0 & 1 & 1 & 0 & 0
\end{array}\right]
$$


The Minimum Total Edge Dominating Energy of a Graph

The characteristic polynomial of $A_{E d}(G)$ is $-\lambda^{7}+\lambda^{6}+14 \lambda^{5}+12 \lambda^{4}-20 \lambda^{3}-28 \lambda^{2}-$ $8 \lambda$ and the characteristic equation is $-\lambda^{7}+\lambda^{6}+14 \lambda^{5}+12 \lambda^{4}-20 \lambda^{3}-28 \lambda^{2}-$ $8 \lambda=0$. The minimum total edge dominating eigenvalues are $\lambda_{1}=0, \lambda_{2}=-1, \lambda_{3}=$ $-2, \lambda_{4}=\sqrt{2}, \lambda_{5}=-\sqrt{2}, \lambda_{6}=2+\sqrt{6}$ and $\lambda_{7}=2-\sqrt{6}$

Therefore the minimum total edge dominating energy of the graph is give by

$$
\begin{gathered}
E_{E d}(G)=|0|(1)+|-1|(1)+|-2|(1)+|\sqrt{2}|(1)+|-\sqrt{2}|(1) \\
+|2+\sqrt{6}|(1)+|2-\sqrt{6}|(1) \\
=3+2 \sqrt{2}+(\sqrt{6}+2)+(2-\sqrt{6}) \approx \mathbf{9 . 8 2 8 4} .
\end{gathered}
$$

Example 2.3. Let $G$ be the complete bipartite $K_{3,3}$ Then the minimum total edge dominating set, $D=\left\{e_{2}, e_{4}, e_{9}\right\}$ where $e_{2}, e_{4}, e_{9}$ are adjacent to each other Therefore

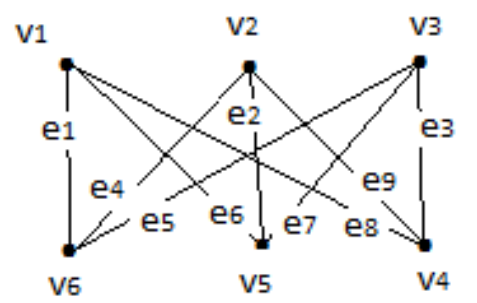

Figure 2: Complete bipartite graph $K_{3,3}$

$$
A_{E d}=\left[\begin{array}{lllllllll}
0 & 0 & 0 & 1 & 1 & 1 & 0 & 1 & 0 \\
0 & 1 & 0 & 1 & 0 & 1 & 1 & 0 & 1 \\
0 & 0 & 0 & 0 & 1 & 0 & 1 & 1 & 1 \\
1 & 1 & 0 & 1 & 1 & 0 & 0 & 0 & 1 \\
1 & 0 & 1 & 1 & 0 & 0 & 1 & 0 & 0 \\
1 & 1 & 0 & 0 & 0 & 0 & 1 & 1 & 0 \\
0 & 1 & 1 & 0 & 1 & 1 & 0 & 0 & 0 \\
1 & 0 & 1 & 0 & 0 & 1 & 0 & 0 & 1 \\
0 & 1 & 1 & 1 & 0 & 0 & 0 & 1 & 1
\end{array}\right]
$$

The characteristic polynomial of $A_{E d}\left(K_{3,3}\right)$ is

$$
-\lambda^{9}+3 \lambda^{8}+15 \lambda^{7}-29 \lambda^{6}-72 \lambda^{5}+108 \lambda^{4}+140 \lambda^{3}-180 \lambda^{2}-96 \lambda+112
$$

And the characteristic equation is

$$
-\lambda^{9}+3 \lambda^{8}+15 \lambda^{7}-29 \lambda^{6}-72 \lambda^{5}+108 \lambda^{4}+140 \lambda^{3}-180 \lambda^{2}-96 \lambda+112=0
$$

. The minimum total edge dominating eigen values are $\lambda_{1}=1, \lambda_{2}=-2, \lambda_{3}=-2$, $\lambda_{4}=3+\sqrt{2}, \lambda_{5}=3-\sqrt{2}, \lambda_{6}=\sqrt{2}, \lambda_{7}=-\sqrt{2}, \lambda_{8}=\sqrt{2}, \lambda_{9}=-\sqrt{2}$,

Therefore the minimum total edge dominating energy of $K_{3,3}$ is give by

$$
\begin{aligned}
& E_{E d}\left(K_{3,3}\right)=|1|(1)+|-2|(1)+|-2|(1)+|\sqrt{2}|(1)+|-\sqrt{2}|(1)+|\sqrt{2}|(1)+ \\
& |-\sqrt{2}|(1)+|3+\sqrt{2}|(1)+|3-\sqrt{2}|(1) \approx \mathbf{1 6 . 6 5 6 8} .
\end{aligned}
$$


Corollary 2.3.1. Minimum total edge dominating energy of a Kuratowski graph $1\left(K_{5}\right)$ is given by $E_{E d}\left(K_{5}\right) \approx \mathbf{2 0 . 2 7 1 5}$.

\section{Minimum total edge dominating energy of some standard graphs}

Theorem 3.1. If $K_{1, p-1}$ is a star with $p \geq 3$ vertices, then

$$
E_{E d}\left(K_{1, p-1}\right)=(p-3)+\sqrt{p^{2}-4 p+8}
$$

Proof: Let $K_{1, p-1}$ be a star graph with vertex set $V=\left\{v_{1}, v_{2}, \ldots \ldots . v_{p}\right\}$. The minimum total edge dominating set of $K_{1, p-1}$ is $D=\left\{e_{1}\right\}$. Then,

$$
A_{E d}\left(K_{1, p-1}\right)=\left[\begin{array}{cccccc}
1 & 1 & 1 & & 1 & 1 \\
1 & 0 & 1 & \cdots & 1 & 1 \\
1 & 1 & 0 & & 1 & 1 \\
& \vdots & & \ddots & & \vdots \\
1 & 1 & 1 & \ldots & 0 & 1 \\
1 & 1 & 1 & & 1 & 0
\end{array}\right]_{p \times p}
$$

The characteristic polynomial of $A_{E d}\left(K_{1, p-1}\right)$ is

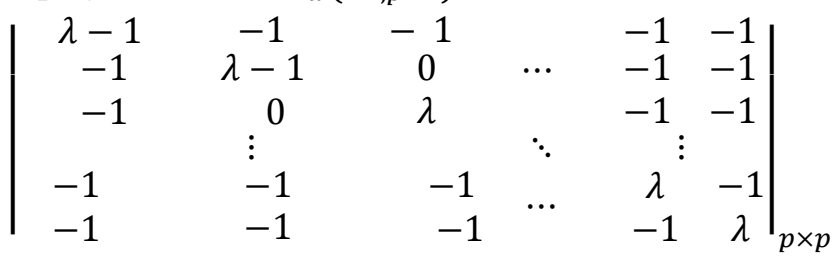

The characteristic equation is $(\lambda+1)^{p-3}\left\{\lambda^{2}-(p-2) \lambda-1\right\}=0$.

The minimum total edge dominating eigenvalues are

$$
\begin{aligned}
\{\lambda=-1(p-3) \text { times }\},\left\{(p-2)+\sqrt{p^{2}-4 p+8}\right\}, \\
\left\{(p-2)+\sqrt{p^{2}-4 p+8}\right\}, \text { (one time each) }
\end{aligned}
$$

Therefore the minimum total edge dominating energy of $\left(K_{1, p-1}\right)$ is

$$
\begin{aligned}
E_{E d}\left(K_{1, p-1}\right)= & |-1|\left\{(p-3) \text { times }+\left\{\sqrt{p^{2}-4 p+8}\right\}+\left\{\sqrt{p^{2}-4 p+8}\right\}\right. \\
& =\{p-3\}+\left\{\sqrt{p^{2}-4 p+8}\right\} .
\end{aligned}
$$

Theorem 3.2. If $K_{p}$ is a complete with $p \geq 3$ vertices, then

$$
E_{E d}\left(K_{p}\right) \approx E_{E d}\left(K_{3}\right)(2.34)^{p-3} \text { with } E_{E d}\left(K_{3}\right) \approx \mathbf{3 . 8 2 8 4}
$$

Proof: Let $K_{p}$ be a complete graph with vertex set $V=\left\{v_{1}, v_{2}, \ldots \ldots v_{p}\right\}$. The minimum total edge dominating set of $K_{p}$ be $D=\left\{e_{1}\right\}$. Then, For $p=3$, We have,

$$
A_{E d}\left(K_{3}\right)=\left[\begin{array}{lll}
1 & 1 & 1 \\
1 & 0 & 1 \\
1 & 1 & 0
\end{array}\right]
$$

The characteristic polynomial of $A_{E d}\left(K_{3}\right)=\left|\begin{array}{ccc}\lambda-1 & -1 & -1 \\ -1 & \lambda & -1 \\ -1 & -1 & \lambda\end{array}\right|$

The characteristic equation is $(-\lambda-1)\left\{\lambda^{2}-2 \lambda-1\right\}=0$

The minimum total edge dominating eigenvalues are

$$
\{\lambda=1 \text { one time }\},(1-\sqrt{2}) \text { and }(1+\sqrt{2}) \text { \{one time each }\}
$$

Therefore the minimum total edge dominating energy of $\left(K_{3}\right)$ is 
The Minimum Total Edge Dominating Energy of a Graph

$$
E_{E d}\left(K_{3}\right)=|1|\{(1)+|1-\sqrt{2}|(1)+|1+\sqrt{2}|(1) \approx \mathbf{3 . 8 2 8 4} .
$$

4. Graphical analysis of minimum total edge dominating energy of $K_{1, p-1}$ and $K_{p}$ Energy of a graph is along $\mathrm{y}$-axis and p-values (number of vertices) are along $\mathrm{x}$-axis

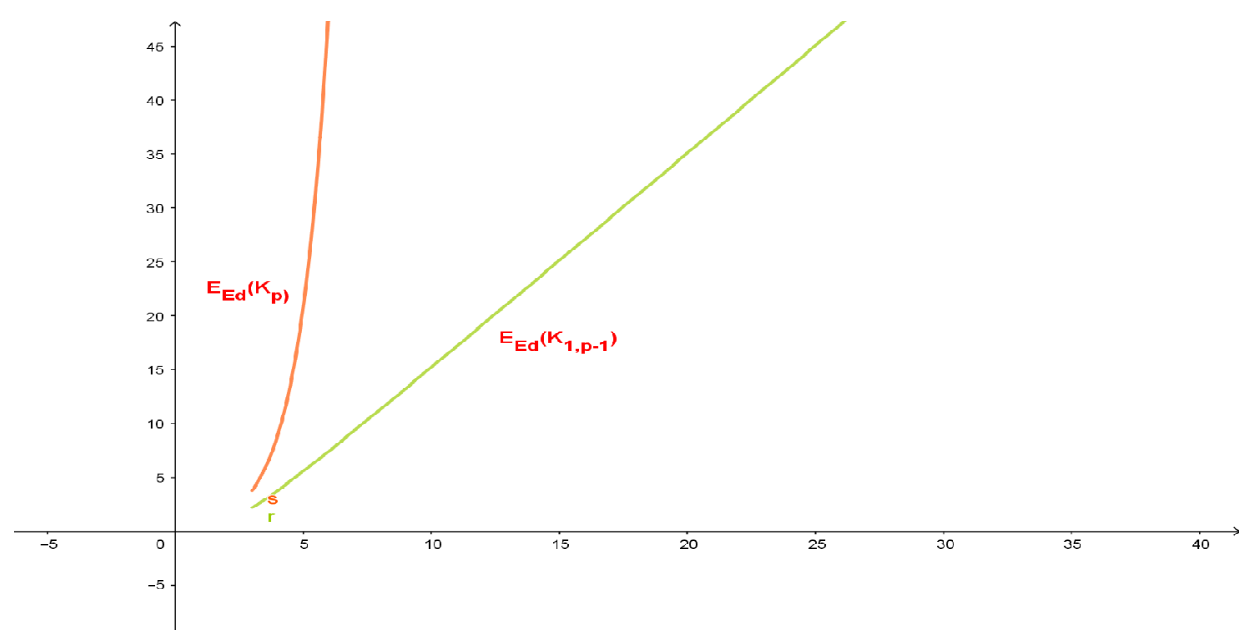

Figure 3: Graph for minimum total edge dominating of complete graph and star graph

The minimum total edge dominating energy of complete graph always greater than the minimum total edge dominating energy of star graph for any value of $p$

\section{Conclusion}

In this paper, the new energy namely Minimum total edge dominating energy is defined and has been found for Star graph and complete graph. The minimum total energy of several other family of graph is an open problem.

Acknowledgement. The authors are highly grateful to the reviewers for their valuable comments for improvement of the paper.

\section{REFERENCES}

1. C.Adiga, A.Bayad, J.Gutman and S.A.Srinivas, The minimum covering energy of a graph, Kragujevac J. Sci., 34 (2012) 39-56.

2. G.Indulal, I.Gutman and Vijaykumar, On distance energy of graph, MATCH Commun. Math. Comput. Chem., 60 (2008) 461-472.

3. G.Rajashekar and A.Venkatesan, Bounds of location-2-Domonation number for product of graphs, Annals of Pure and Applied Mathematics,15(1) (2017) 1-11

4. I.Gutman, The energy of a graph, Ber. Math. Statist. Sket. for Schungsz, Graz, 103 (1978) 1-22.

5. I.Gutman and B.Zhou, Laplacian energy of a graph, Lin, Algebra Appl., 414 (2006) 29-37. 
6. M.R.Joo, D.Yandeh and M.Kiani, Mirzakhah, Incidence energy of a graph MATCH Commun. Math. Comput. Chem., 62 (2009) 561-572.

7. M.R.Rajesh Kanna, B.N.Dharmndra and G.Sridhara, The Minimum dominating energy of a graph, International Journal of Pure and Applied Mathematics, 85 (4) (2013) 707-718.

8. S.Meenakshi and S.Lavanya, A survey on energy of a graph, Annals of Pure and Applied Mathematics, 8(2) (2014) 183-234.

9. V.R.Kulli, Theory of domination in graphs, Vishwa International Publications, Gulbarga, India, 2010. 